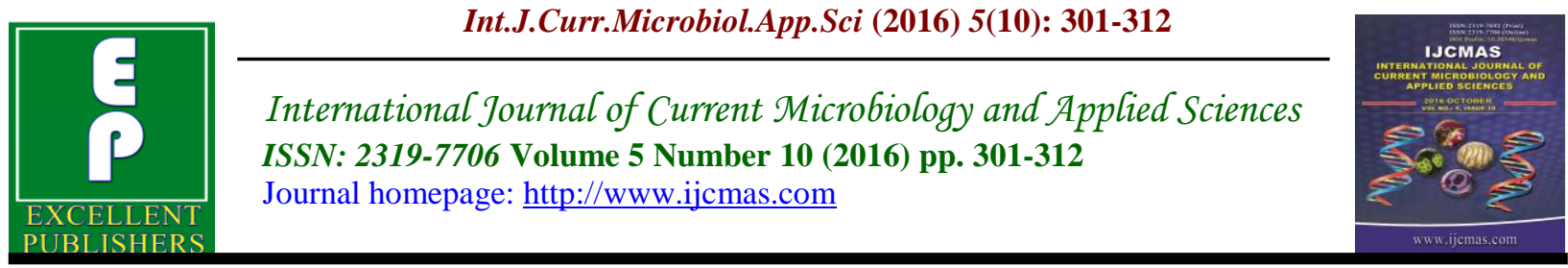

Original Research Article

http://dx.doi.org/10.20546/ijcmas.2016.510.034

\title{
Use of Pseudomonas aeruginosa as Fertilizer in Eruca sativa
}

\author{
Areej Ali Baeshen* \\ Department of Biological Sciences, Faculty of Science, \\ King Abdulaziz University, Jeddah, Saudi Arabia \\ *Corresponding author
}

Keywords

Pseudomonas aeruginosa, fertilizer, Eruca sativa, Pyocyanin, crop plants.

\section{Article Info}

Accepted: 14 September 2016 Available Online: 10 October 2016

\section{A B S T R A C T}

P. aeruginosa is more widely known as an opportunistic pathogen for humans and animals than as soil bacteria, for this reason it was used as fertilizer in many crops as well as against root rot without harmful action. It is Pyocyanin pigment may consider as plant growth promoting, and can act as a cheap source of fertilizers to crop plants. Pyocyanin production by the selected P. aeroginosa isolates L. 12, L.16 and L.17 was determined in different media. The crude Pyocyanin was taken as control and other treatments were done by adding distilled water, Viz., 5\%, 15\% and $25 \%$. E. sativa seedlings were irrigated every other day using these solutions. Measurements of leaf area, biomass Water content, photosynthesis pigments and essential and heavy elements were taken. Results revealed a pronounce increase in low Pyocyanin concentration (5\%) treated plants compared to control in all measured parameter. On the other hand, high Pyocyanin concentration treated plants achieved the highest elements constituents. Pyocyanin may be further improved with the optimization and familiarization according to the current soil conditions. In future, it may replace the chemical fertilizers and help in sustainable agriculture. Further research and understanding of mechanisms of Pyocyanin is urgently needed.

\section{Introduction}

Eruca sativa is widely distributed all over the world and is usually consumed fresh for its special taste. It is a herb of traditional medicine and it contains many ingredients such as glucosinolates vitamin $\mathrm{C}$, carotenoids, and flavonoids (Saleh et al., 2009). Pseudomonas aeruginosa is a common Gram-negative rod-shaped bacterium that can cause disease in plants and animals, including humans.

The direct use of microorganisms to promote plant growth and to control plant pests and diseases continue to be an area of speedily going up research. Many researchers have showed that the positive effect of Pseudomonas aeruginosa on the productivity of many crops in different soils and climatic conditions (Das et al., 2010 and Santos et al., 2013; Bashan et al., (2011) concluded Prosopis articulata, Parkinsonia microphylla, Parkinsonia florida helped in biomass increase of seedlings. In addition, Liu et al., (2013) reported thatRhizobacteria together with fertilizers helped high dry matter accumulation and nutrient uptake in the Fraxinus americana forest seedlings. 
$P$. aeruginosa is more widely known as an opportunistic pathogen for humans and animals than as soil bacteria, for this reason it was used as fertilizer to many crops as well as against root rot without harmful action (Farruk et al., 2007). Instead, Pseudomonas spp. have appeared to be an important biological mean to control plant diseases (Ultan et al., 2001 and Prasada and Paramageetham, 2013).

In this study Pseudomonas aeruginosa species were isolated from forest litter and evaluated for its bio control activity against Sclerotium rolfsii Sacc. Horticulture is one of the areas where agricultural technologies are widely and intensively used. Horticultural crops may grow both in the open and closed space such as greenhouse and tunnel, and there are several practical applications such as propagation with cuttings and grafting, pruning, and soilless culture; plant growth regulators that have little or no use with other agricultural crops were used largely in horticultural crop production (Ahmet, 2011).

\section{Research Methodology}

\section{Bacterial isolates}

Pseudomonas aeruginosa isolates used in the study

Identified Pseudomonas aeruginosa (20 isolates) were obtained from a previous study, which was collected from contact lens storage cases, contact lenses and contact lens wearer in Saudi Arabia (Salha, 2012).

\section{Preservation of the used organisms}

Pseudomonas aeruginosa isolates were maintained on cetrimide agar at $4^{\circ} \mathrm{C}$, while tested bacteria were maintained on slopes of Nutrient agar at $4^{\circ} \mathrm{C}$. All the tested fungi maintained on slopes of Sabouraud medium at $4^{\circ} \mathrm{C}$. All the organisms were regenerated every six months, according to Dadgar et al. (2006).

Screening the ability of $P$. aeroginsa to produce pigments on Cetrimide agar

All isolates of Pseudomonas aeruginosa were screened for pigments production on cetrimide agar medium. After preparing the medium, it was poured in sterile condition on Petri dishes and left to solidified. All the bacterial isolates were incubated on the previous medium at $35^{\circ} \mathrm{C}$ for 3 days.

Effect of cultural conditions on the production of pyocyanin by selected isolates of $P$. aeruginosa

These experiments were performed in $250 \mathrm{ml}$ Erlenmeyer flasks containing $48 \mathrm{ml}$ of the production medium, inoculated with $2 \mathrm{ml}$ of the overnight culture of the selected $P$. aeruginosa isolate L.12, L.16 or L.17. The flasks were incubated at $35^{\circ} \mathrm{C}$ for 6 days. At the end of the incubation period, the cells were collected by centrifugation at $4000 \mathrm{rpm}$ for $30 \mathrm{~min}$ and the quantity of pyocyanin was measured as described before.

Effect of different media on the production pyocyanin by selected isolates of $P$. aeruginosa

Pyocyanin production by the selected $P$. aeroginosa isolates L. 12, L.16 and L.17 was determined in different media. The media used were King's medium B Base (King et al., 1954). King's Medium A Base (King et al., 1954), Pseudomonas F medium (King et al., 1954), Pseudomonas P medium (Forbes et al., 2007). After preparation of these different media in 250 $\mathrm{ml}$ Erlenmeyer flasks containing $48 \mathrm{ml}$ of the media, they were inoculated with $2 \mathrm{ml}$ of the selected bacterial isolate L.12, L.16 or L.17 and the flasks were incubated at $35^{\circ} \mathrm{C}$. After 6 days of incubation period. 


\section{Applications of pyocyanin pigment}

The crude pyocyanin was taken as control and other treatments were done by adding distilled water, Viz., 5\%, $15 \%$ and $25 \%$. E. sativa seedlings were irrigated every other day using these solutions.

\section{Measurements}

One month later, many parameters have been estimated. These include leaf area, root and shoot lengths, biomass, water content, photosynthetic pigments, in addition to the amount of essential and heavy elements in shoots and roots.

\section{Statistical Analysis}

The results were analyzed statistically by using the SPSS BASE 20.0 for windows (SPSS Inc., Chicago, IL) packages. Data were tested by ANOVA and means were analyzed at $P \leqslant 0.05$ and $P \leqslant 0.01$ levels of significant, to determine the significant differences between different treatments.

\section{Results and Discussion}

Leaf is of $E$. sativa treated with different concentrations of pyocyanin

E. sativa leaf areas showed different responses when treated with different concentrations of pyocyanin pigment as was shown in Fig 1. Leaf areas were high in all treated plants compared to control. The increase followed the pattern 5\% Pyocyanin $\left(8.9 \mathrm{~cm}^{2}\right)>15 \%$ Pyocyanin $\left(7.8 \mathrm{~cm}^{2}\right)>25 \%$ Pyocyanin $\left(6.7 \mathrm{~cm}^{2}\right)$. The control plants recorded the lowest leaf area $\left(3.5 \mathrm{~cm}^{2}\right)$.

Biomass of $E$. sativa treated with different concentrations of pyocyanin

The maximum growth in the shoots and roots lengths was observed principally in shoots and roots of the treated plants across all treatments (Fig.2). The above and below ground lengths increase in different treatments was in the following order: 5\% Pyocyanin > $15 \%$ Pyocyanin)> $25 \%$ Pyocyanin. $(40 \mathrm{~cm}, 23.5 \mathrm{~cm}, 23 \mathrm{~cm}$ in shoot) and $(25 \%>5 \%>15 \%$ in roots. $(10 \mathrm{~cm}, 9.3 \mathrm{~cm}$ and $8.3 \mathrm{~cm}$, respectively). The control plants recorded the lowest shoot and root lengths (14.4 $\mathrm{cm}$ and $7.3 \mathrm{~cm}$, respectively).

\section{Water Content of $E$. sativa Shoots and roots treated with different concentrations of Pyocyanin}

Fig. 3 illustrates the amount of water content of shoots and roots of $E$. sativa treated with different concentrations of Pyocyanin. There was no pronounce effect in water content of shoots, nonetheless, control plants recorded the highest value $(92.3 \%)$ but there is no significant difference compared to treated plants. On the other hand, treated plants showed high water content compared to control, especially $5 \%$ concentration $(76.7 \%$ compared to $60.7 \%$ of the control.

Photosynthesis Pigments of $E$. sativa treated with different concentrations of Pyocyanin

In general, photosynthesis pigments showed different response to the treatments. Regardless of the treatments, all plants recorded higher amounts of carotenoides followed by chlorophyll $\mathrm{A}$ and less amounts of chlorophyll B (Fig. 4). Treated plants (5\% and $15 \%$ ) achieved the highest amount of chlorophyll A (1.29 $\mu \mathrm{mol} / \mathrm{g} /$ fresh wt.), followed by $25 \%$ treatment (1.02 $\mu \mathrm{mol} / \mathrm{g} /$ fresh wt), while the control plants recorded the lowest amount of chlorophyll A $(0.26 \mu \mathrm{mol} / \mathrm{g} /$ fresh wt $)(P \leq 0.01)$.

The same pattern was observed in the amount of chlorophyll B and the carotenoids. All treated plants recorded high 
amount of chlorophyll B and carotenoids, while control plants showed the lowest amount of these pigments (Fig. 4). Worth mentioning, $5 \%$ treatment recorded 5 times and 6 times more of chlorophyll $\mathrm{B}$ and carotenoids $(0.52 \mu \mathrm{mol} / \mathrm{g} /$ fresh wt compared to $0.099 \mu \mathrm{mol} / \mathrm{g} /$ fresh $\mathrm{wt}$ ) for chlorophyll B and $(1.59 \mu \mathrm{mol} / \mathrm{g} /$ fresh wt compared to 0.26 $\mu \mathrm{mol} / \mathrm{g} /$ fresh wt) for carotenoides.

\section{Amount of Essential and Heavy Elements of $E$. sativa Shoots treated with different concentrations of Pyocyanin}

Essential elements such as $\mathrm{Na}, \mathrm{Mg}, \mathrm{C}, \mathrm{K}, \mathrm{P}$, $\mathrm{N}$ and $\mathrm{Cl}$, (Fig. 5), showed different trends as a response to treatment with different concentration of pyocyanin. For instance, $5 \%$ plants had the lowest amount of sodium while the control recorded the highest value of sodium. On the other hand, high accumulation of $\mathrm{Ca}, \mathrm{K}$ and $\mathrm{N}$, in the aerial parts of E. sativa shoots were recorded in $15 \%$ treated plants and lowest values in control. Concerning $\mathrm{P}$, there were no significant differences among treatments or with the control plants. Accumulation of heavy elements in shoots of E. sativa, followed different trends than essential ones (Fig. 6). All plants had low amount of $\mathrm{Pb}$, and $\mathrm{Cu} .15 \%$ treated plants accumulated large amounts of B, $\mathrm{Zn}$ and Fe. On the other hand, 5\% treated plants accumulated almost the same amount of $\mathrm{Mn}$ as the control plants, while $15 \%$ and $25 \%$ treated plant recorded almost the same amount of Mn.

\section{Amount of Essential and Heavy Elements of $E$. sativa Roots treated with different concentrations of Pyocyanin}

Fig. 7 and 8 illustrated the amount of essential and heavy elements accumulated in roots of treated and control E.sativa plant. Fig 7 indicated that 5\% treated plants recorded less essential elements in terms of $\mathrm{Na}, \mathrm{Mg}, \mathrm{Ca}, \mathrm{P}$ and $\mathrm{N}$. In contrast, $15 \%$ and
$25 \%$ accumulated large amount of $\mathrm{Na}, \mathrm{Mg}$, $\mathrm{Ca}, \mathrm{K}, \mathrm{P}$ and $\mathrm{N}$. control plants however recorded moderate amounts of these elements in roots. Regarding heavy elements (Fig. 8), control plants recorded the highest amount of $\mathrm{Pb}, \mathrm{B}, \mathrm{Cu}, \mathrm{Mn}$ and $\mathrm{Fe} .5 \%$ treated plants accumulated the least amount of $\mathrm{Pb}$, $\mathrm{Zn}$ and $\mathrm{Mn}$, while $15 \%$ treated plants recorded the highest amount of $\mathrm{Zn}$. $25 \%$ plants on the other hand, accumulated large amount of $\mathrm{Pb}$ and $\mathrm{Mn}$. Worth mentioning is that, the trend of Fe was as follows, control plants $>5 \%$ treated plants $>15 \%$ treated plants $>25 \%$ treated plants.

P. aeruginosa low concentration of Pycyanin isolated in this study seemed to have positive effects on plant growth to encourage the growth and development of $E$. sativa in many ways. For Instance, leaf area increase by almost two and a half times at $5 \%$ concentration compared to the control. Leaf area growth reveals light interception and considered as an main factor in determining plant productivity (Gifford et al., 1984; Koester et al., 2014).

Moreover, high performance of plants frequently depends on optical methods in which leaf area growth is compared with estimates of photosynthesis for plants with altered photosynthetic rates because they are non-destructive and cost-effective (Golzarian et al., 2011; Zhang et al., 2012; Tessmer et al., 2013 and Radhapriya et al., 2015). Concerning shoot and root lengths, $5 \%$ Pyocyanin concentration pigment achieved E.sativa to record high values compared to control. Moreover, all treated plants showed high shoot and root lengths compared to the control.

The motivation of growth traits of E.sativa caused by Pyocyanin pigment was clear in the biomass of this plant in terms of shoot and root fresh and dry weights. All treated plants recorded the highest biomass in the 
manner 5\%> 15\%> 25\%. The appliance of $P$. aeruginosa strains is known to improve the shoot, root, and panicle growth as well as plant height in the case of tomato plants (Sabin and Tehmina, 2016). Miransari, (2011) reported that this increase in plant growth occurs because of the production of growth related substances. Moreover, wheat, P.aeruginosa caused increased germination of seeds and plant shoot and root as was reported by Arif et al., (2015).

Many researchers concluded that this increase in shoot and root growth is mainly due to the fact that, these bacteria products act directly on plant roots through the production of indole acetic acid (IAA) (Cheryl and Bermand, 2002). This phytohormone is supposed to stimulate the formation of lateral roots and increase nutrient absorption from root hairs (Sisir and Basu, 2006), and is known to stimulate the cell elongation and cell division of plant roots (Farah et al., 2004).

In addition, some authors reported that, chemical fertilization of seedlings with commercial biofertilizer resulted in a significant increase in biomass when compared with the control but was shown to be less effective than the combined application of $P$. aeruginosa RRALC3 and chemical fertilizer (Vessey, 2003).

Water contents of treated Esativa shoots showed no significant effect when compared to control. However, roots of treated plants recorded high amount of water contents especially 5\% treatment compared to control. Researchers concluded that plant root are potent growth signals and vital because of their influence on nutrient acquirement by plants (Travis et al., 2004 and Besharat et al., 2010).

Fig.1 Leaf area (Cm2) of E. sativa and shoot and Root lengths treated with different concentration of $P$. aeruginosa pigments

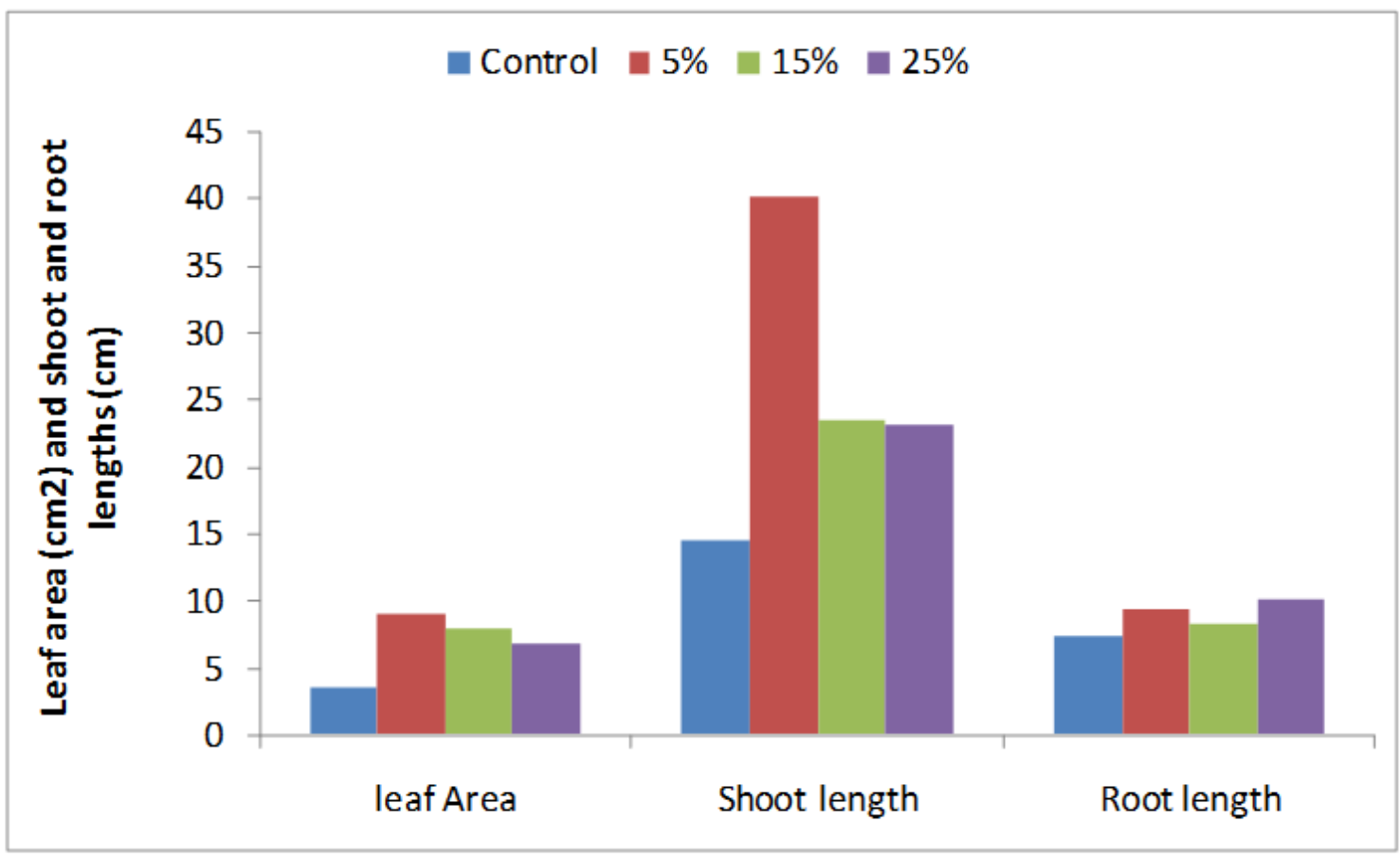


Fig.2 Biomass (g) of E. sativa treated with different concentration of $P$. aeruginosa pigments.

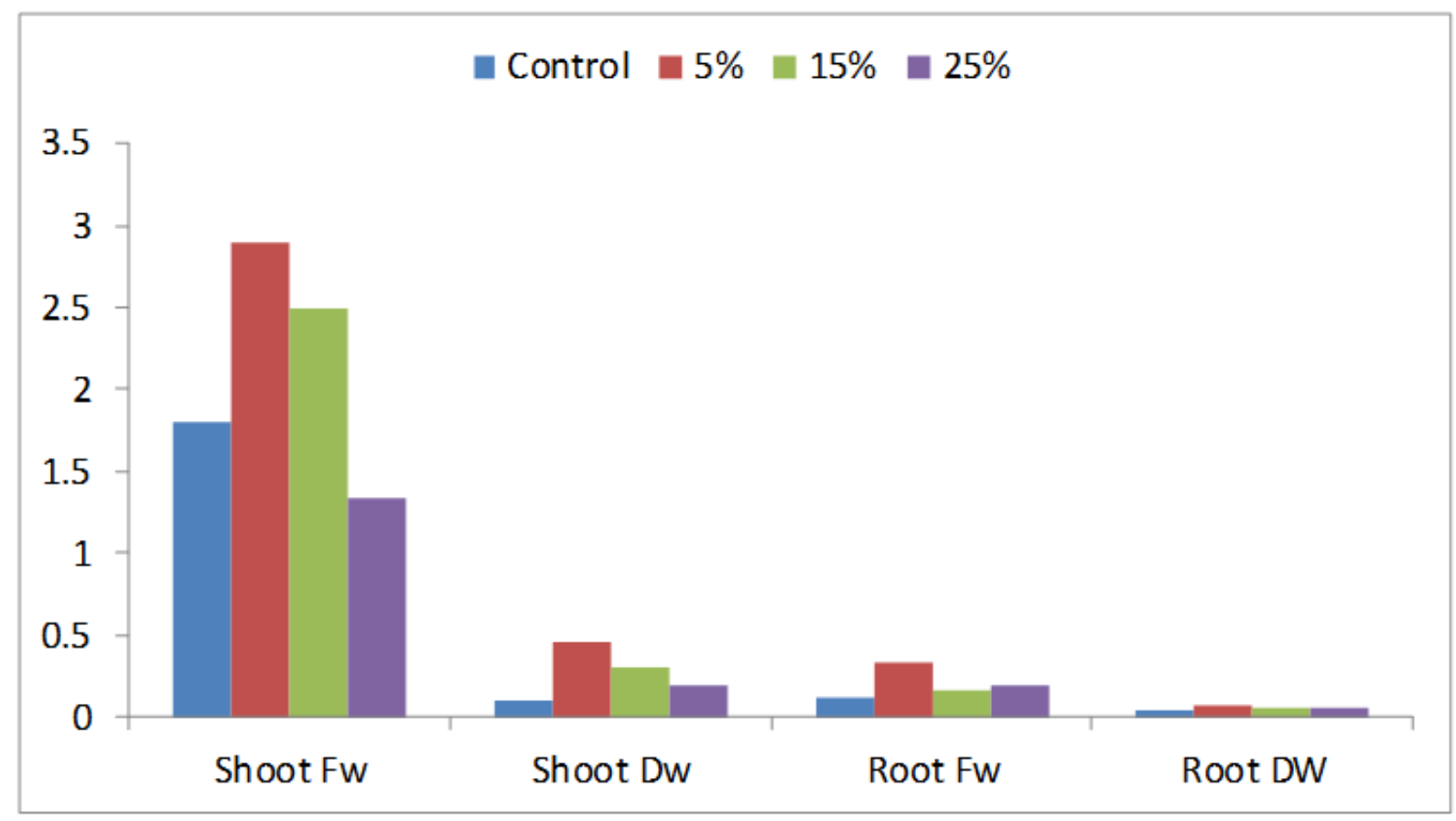

Fig.3 water Content (\%) of E. sativa treated with different concentration of $P$. aeruginosa pigments

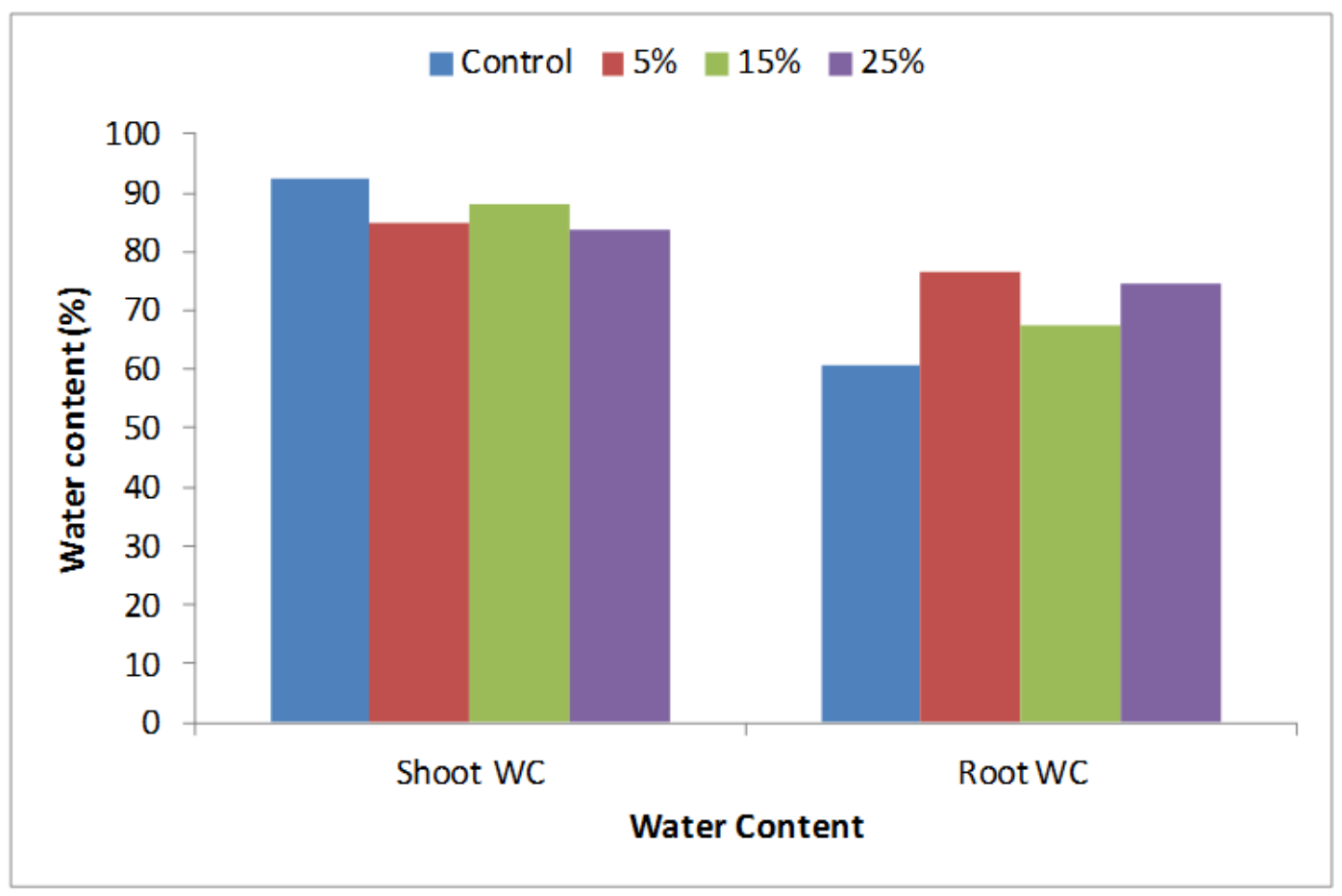


Fig.4 Photosynthetic Pigments ( $\mu \mathrm{mol} / \mathrm{g} /$ fresh wt.) of E. sativa treated with different concentration of $P$. aeruginosa pigments

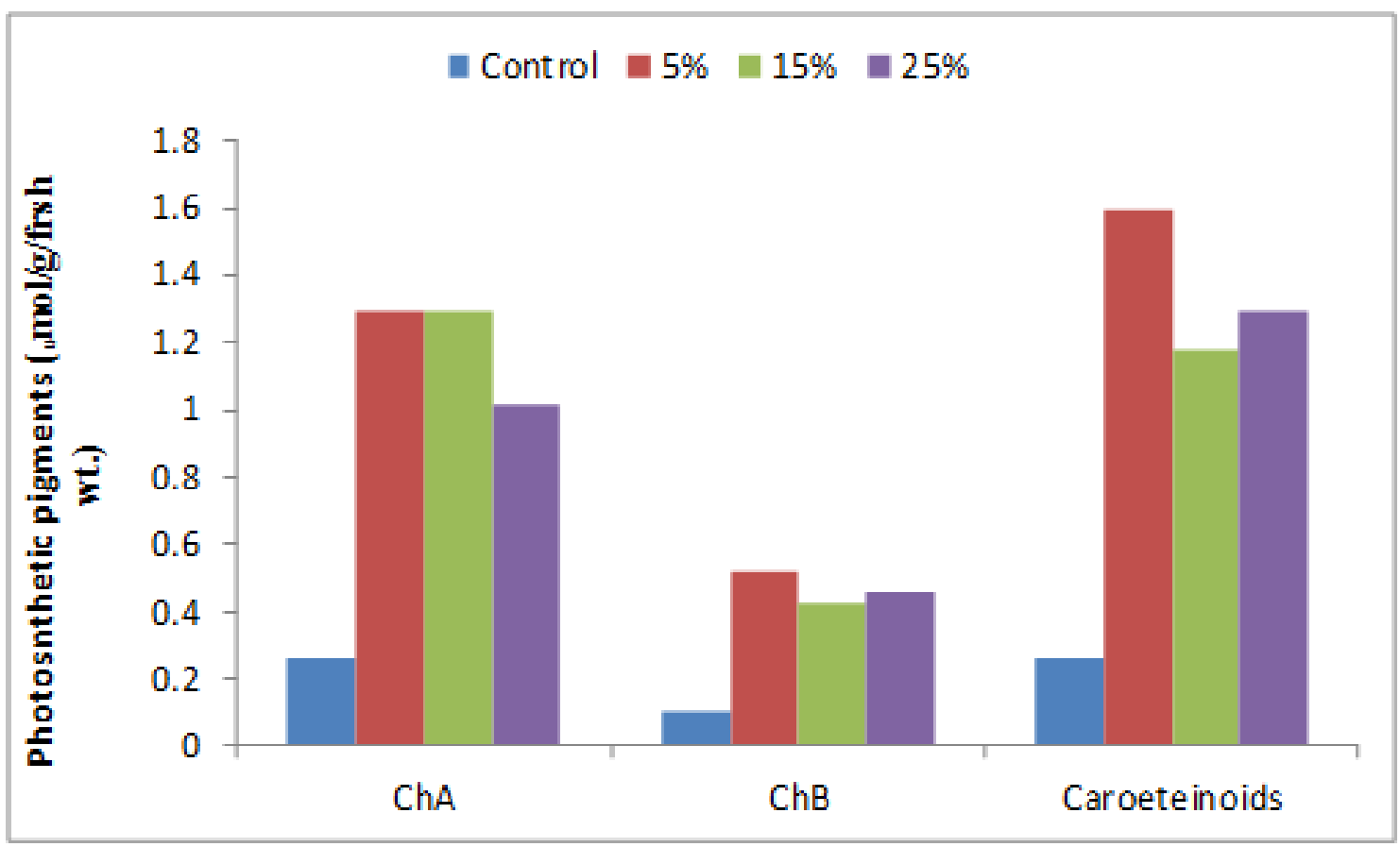

Fig.5 Amount of Essential Elements (\%) of E. sativa Shoots treated with different concentration of $P$. aeruginosa pigments

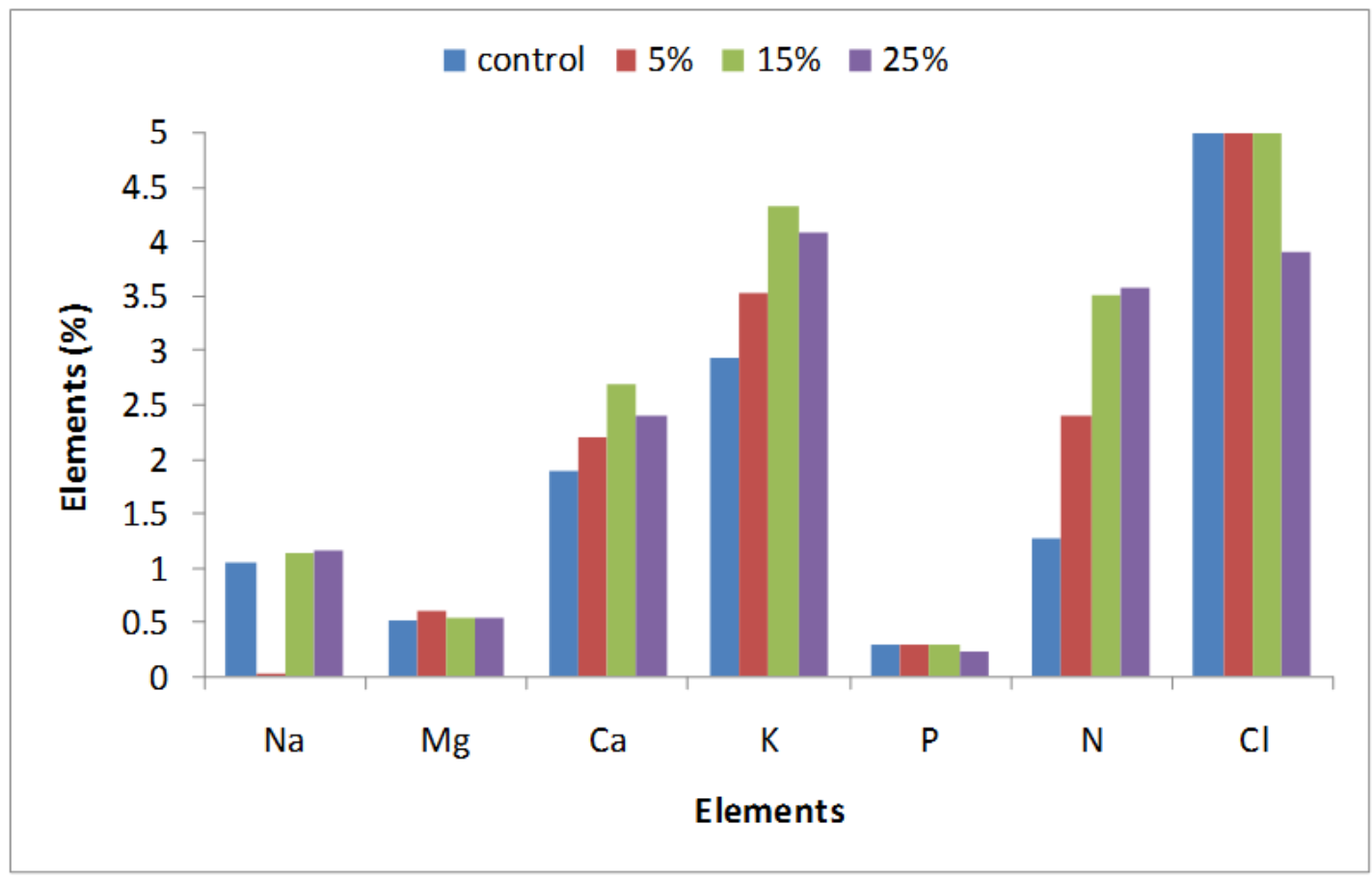


Fig.6 Amount of Heavy Elements (ppm) of E. sativa Shoots treated with different concentration of $P$. aeruginosa pigments

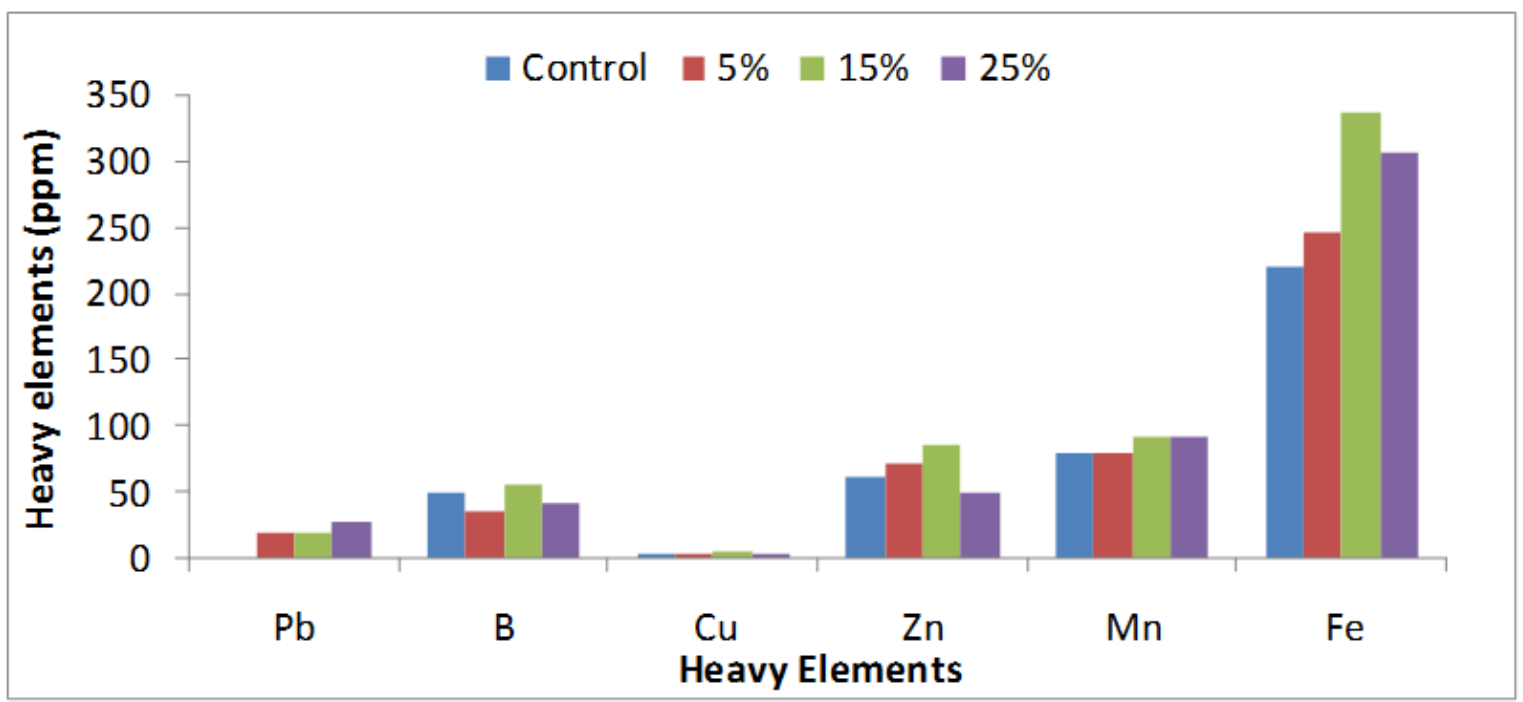

Fig.7 Amount of Essential Elements (\%) of E. sativa Roots treated with different concentration of $P$. aeruginosa pigments

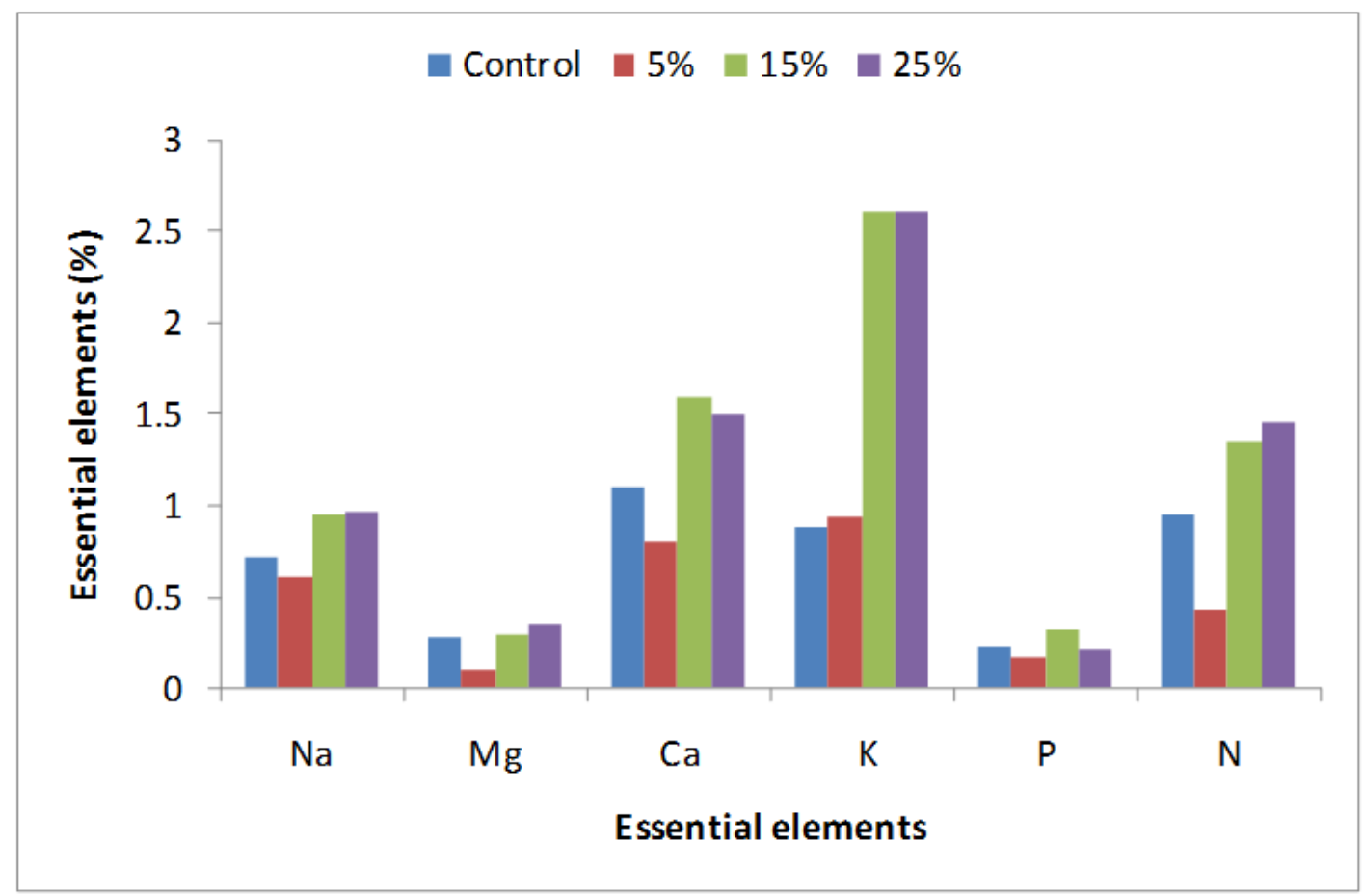


Fig.8 Amount of Heavy Elements (ppm) of E. sativa Roots treated with different concentration of $P$. aeruginosa pigments

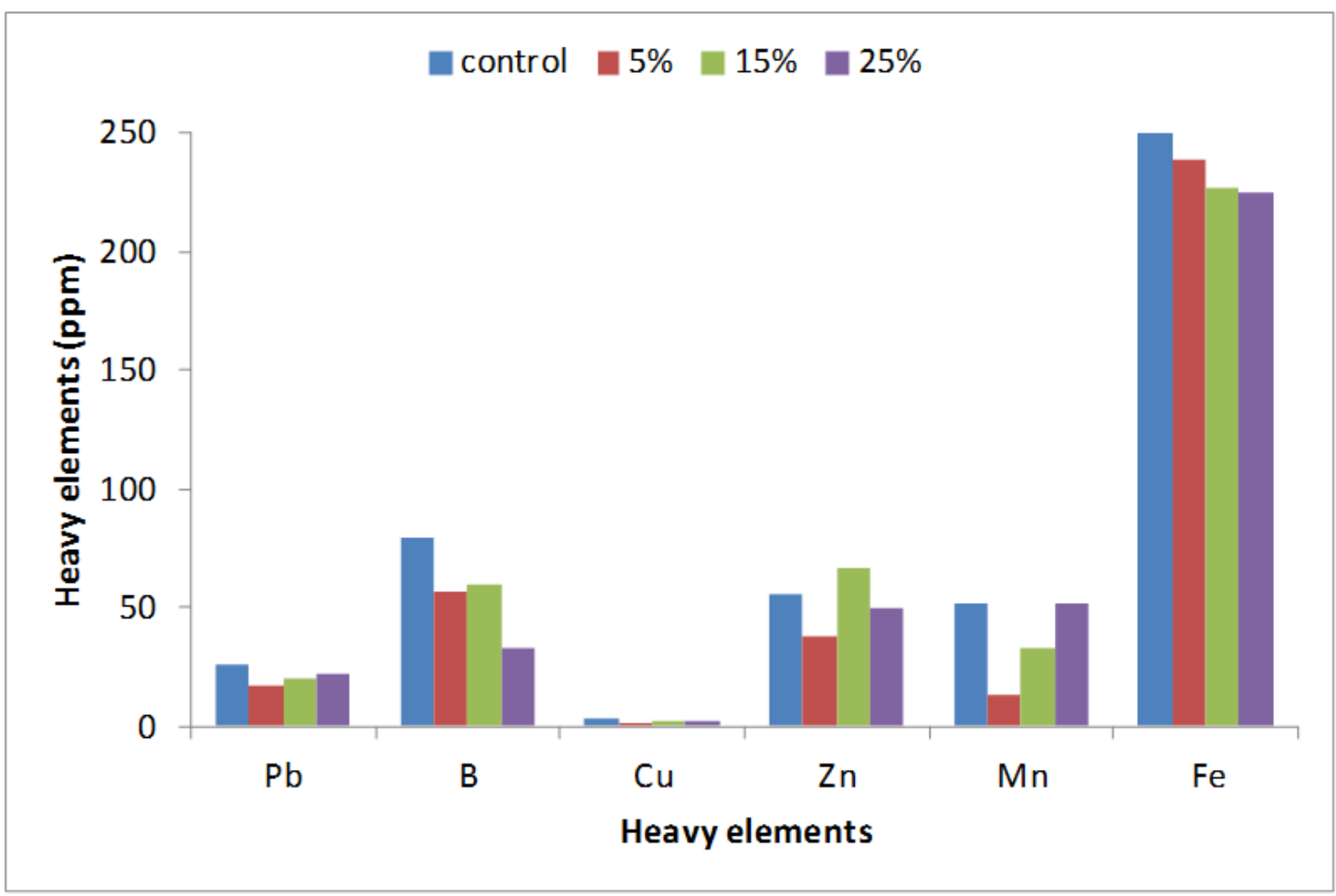

Photosynthesis pigments, in terms of chlorophyll A and carotenoids showed pronounce increase in treated plants compared to control. Increased photosynthesis pigment with Pseudomonas treatment was reported by Islam et al., (2014) in wheat. In general, the beneficial effect of high photosynthesis pigments is well known fact (Lawlor, 2002 and Chaves et al., 2009).

Element accumulation in shoots revealed that, 5\% treatment recorded low $\mathrm{Na}$ amount while the control plants recorded the highest value. In general low concentrations recorded the fewer amounts in case of essential and heavy elements, while the high concentrations accumulated more amounts of these elements.

Additionally, in the present study, it was found that high concentration treatment of
Pyocyanin (15\% and 25\%) increased the $\mathrm{Na}$, $\mathrm{Mg}, \mathrm{K}, \mathrm{Ca}$ and $\mathrm{Cl}$ content of $E$. sativa seedlings roots. This might be credited to the production of organic acids by the plants themselves as well as by various bacteria in the rhizosphere because production of organic acids is influential in decreasing the soil $\mathrm{pH}$ and rousing the accessibility of micronutrients as was reported by Berg (2009). Regarding heavy elements, low concentration $(5 \%)$ treated plants accumulated less $\mathrm{Pb}, \mathrm{Zn}$ and $\mathrm{Mn}$, while the other treatments recorded high amount of these elements. It was noticeable that $\mathrm{Fe}$ was abundant in shoots and roots of treated plants. This considered a good factor for the growth of E. sativa. Iron is a vital nutrient for almost all forms of life. All microorganisms essentially require iron (Neilands, 1995). In the aerobic environment, iron come up primarily as $\mathrm{Fe}^{3+}$ and is likely to form insoluble 
hydroxides and oxyhydroxides, thus making it commonly unreachable to both plants and microorganisms (Rajkumar et al., 2010).

In conclusion, plant growth promoting Pyocyanin, may act as a cheap source of fertilizers to crop plants. The productive competence may be further improved with the optimization and familiarization according to the current soil conditions. In future, they are expected to replace the chemical fertilizers and help in sustainable agriculture. Further research and understanding of mechanisms of Pyocyanin is urgently needed.

\section{References}

Ahmet, E. 2011. Use of Plant Growth Promoting Rhizobacteria in Horticultural crops. Bacteria in Agrobiology: Crop Ecosystems, pp 189-235. Publisher Springer Berlin Heidelberg.

Arif, F., Silini, A., Cherif, H. and Ghoul, M. 2015. Plant Growth Promoting and Antagonistic Traits of Indigenous Fluorescent Pseudomonas SPP. Isolated from Wheat Rhizosphere and a. Halimus Endoshere. European Scientific J., vol.11, No.24 ISSN: 1857 $-7881$.

Bashan, Y., SalaZar, B.G., Moreno, M., Lopez, B.R., Linderman, R.G. 2012. Restoration of eroded soil in the Sonoran desert with native leguminous trees using plant growth-promoting microorganisms and limited amounts of compost and water. J. Environ. Manage., 102: 26-36. doi: 10.1016/j.jenvman. 2011.12.032. pmid:22425876.

Berg, G. 2009. Plant-microbe interactions promoting plant growth and health: perspectives for controlled use of microorganisms in agriculture. Appl.
Microbiol. Biotechnol., 84: 11-18.

Besharat, S., A.H. Nazemi, A.A. Sadraddini 2010. Parametric modeling of root length density and root water uptake in unsaturated soil. Turkey J. Agri., Vol. 34: 439-449.

Chaves, M.M., Flexas, J., and Pinheiro, C. 2009. Photosynthesis under drought and salt stress: regulation mechanisms from whole plant to cell. Ann. Bot., 103: 551-560. doi: 10.1093/aob/mcn 125 .

Cheryl, L.P. and Bernard, R.G. 2002. Role of Pseudomonas putida Indole acetic Acid in Development of the Host Plant Root System. Appl. Environ. Microbiol., 68(8): 3795-3801.

Dadgar, T., Asmar, M., Saifi, A., Mazandarani. M., Bayat, H., Moradi, A., Bazueri, M. and Ghemi, E. 2006. Antibacterial activity of certain Iranian nedicinal plants against MethicillinResistant and sensitive Staphylococcus aureus, Asian J. Plant Sci., vol. 5 (5): 861-866.

Das, S.N., Dutta, S., Kondreddy, A., Chilukoti, N., Pullabhotla, S.V.S.R.N., et al. 2010. Plant growth- promoting chitinolytic Paenibacillus elgii responds positively to tobacco root exudates. J. Plant Growth Regul., 29: 409-418. doi: 10.1007/s00344-0109152-1

Farah, A., Iqbal, A. and Mohd, S.K. 2005. Indole Acetic Acid Production by the Indigenous Isolates of Azotobacter and Fluorescent Pseudomonas in the Presence and Absence of Tryptophan. Turkey J. Biol., 29: 29-34.

Farrukh, M., Viqar, S. and Syed, E. 2007. Enhancement of Biocontrol Potential of Pseudomonas aeruginosa and Paecilomyces lilacinus against Root Rot of mungbean by a MedicinalL Plant Launaea nudicaulis L. Pak. J. Bot., 39(6): 2113-2119. 
Forbes, A., Sahm, G. and Weissfeld, S. 2007. Bailey \& Scott's diagnostic microbiology, 12th ed. Mosby, Inc., St. Louis, Mo.

Gifford, R.M., Thorne, J.H., Hitz, W.D., Giaquinta, R.T. 1984. Crop productivity and photo assimilate partitioning. Sci., 24: 801-808.

Golzarian, M.R., Frick, R.A., Rajendran, K., Berger, B., Roy, S., Tester, M., et al. 2011. Accurate inference of shoo $t$ biomass from high-throughput images of cereal plants. Plant Methods, 7: 1122.

Islam, F., Yasmeen, T., Ali, Q., Ali, S., Arif, M.S., Hussain, S., Rizvi, H. 2014. Influenceof Pseudomonas aeruginosa as PGPR on oxidative stress tolerance in wheat under $\mathrm{Zn}$ stress. Ecotoxicol. Environ. Safety, 104: 285-293.

King, E.O., Ward, M.K., Raney, D.E. 1954. Two simple media for the demonstration of Pyocyanin and fluorescent. J. Lab. Clin. Med., 44(2): 301-7.

Koester, R.P., Skoneczka, J.A., Cary, T.R., Diers, B.W., Ainsworth, E.A. 2014. Historical gains in soybean (Glycine max Merr.) seed yield are driven by linear increases in light interception, energy conversion, and partitioning efficiencies. $J$. Experimental Bot., 65: 3311-3321.

Lawlor, D.W. 2002. Limitation to photosynthesis in water-stressed leaves: stomata vs. metabolism and the role of ATP. Ann. Bot., 89: 871-885. doi: 10.1093/aob/mcf110.

Liu, F., Xing, S., Ma, H., Du, Z., Ma, B. 2013. Plant growth-promoting rhizobacteria affect the growth and nutrient uptake of Fraxinus americana container seedlings.

Miransari, M. 2011. Interactions between arbuscular mycorrhizal fungi and soil bacteria. Appl. Microbiol. Biotechnol.,
89: 91.

Neilands, J.B. 1995. Siderophores: structure and function of microbial iron transport compounds. J. Biol. Chem., 270, pp. 26723-26726

Prasada, G.B.1 and Paramageetham, Ch. 2013. Biocontrol of Sclerotium rolfsii - a polyphagous plant pathogen by Pseudomonas aeruginosa isolated from forest litter. Int. J. Res. Plant Sci., 3(1): 1-4.

Radhapriya, P., Ramachandran, A., Anandham, R., Mahalingam, S. 2015. Pseudomonas aeruginosa RRALC3 Enhances the Biomass, Nutrient and Carbon Contents of Pongamia pinnata Seedlings in Degraded Forest Soil. PLoS ONE, 10(10): e0139881. doi:10.1371/journal.pone.0139881

Rajkumar, N., Ae, M.N.V. Prasad, H. Freitas. 2010. Potential of siderophore-producing bacteria for improving heavy metal phytoextraction. Trends Biotechnol., 28, pp. 142-149.

Sabin, F. and Tehmina, A. 2016. Induction of Systemic Resistance in tomato by Pseudomonas aeruginosa PM12 against Fusarium wilts. Pak. J. Biotechnol., Vol. 13(2): 117-124.

Saleh Alqasoumi, Mohammed AlSohaibani, Tawfeq Al-Howiriny, Mohammed Al-Yahya, Syed Rafatullah. 2009. Rocket "Eruca sativa": A salad herb with potential gastric anti-ulcer activity. World $J$. Gastroenterol., 15(16): 1958-1965.

Salha, H.M. 2012. Bacteria isolated from contact and non contact lens and antibiotic susceptibility patterns of isolated Pseudomonas aeruginosa. African J. Microbiol. Res., Vol.6 (47), pp. 7350-7356.

Santos, V., Folter, S., Delano-Frier, J.P., Gomez-Lim, M.A., Guzman-Ortiz, D.A. 2013. Growth promotion and 
flowering induction in Mango (Mangifera indica L. cv "Ataulfo") trees by Burkholderia and rhizobium inoculation:. J. Plant Growth Regul., 32: 615-627. doi: 10.1007/s00344013-9329

Sisir, G., Basu, P.S. 2006. Production and metabolism of indole acetic acid in roots and root nodules of Phaseolus mungo. Microbiol. Res., Volume 161(4): 362-366.

Tessmer, O.L., Jiao, Y., Cruz, J.A., Kramer, D.M., Chen, J. 2013. Functional approach to high-throughput plant growth analysis. BMC Systems Biol., 7: S17.

Travis, S.W., Harsh, P.B., Eric Déziel, Herbert, P.S., Rahme, R.F. and Jorge, M.V. 2004. Pseudomonas aeruginosa-
Plant Root Interactions. Pathogenicity, Biofilm Formation, and Root Exudation. Plant Physiol., vol. 134(1): 320-331.

Ultan, F.W., John, P.M. and Fergal, O.G. 2001. Pseudomonas for biocontrol of phytopathogens: from functional genomics to commercial exploitation. Curr. Opinion in Biotechnol., 12: 289295.

Vessey, J.K. 2003. Plant growth promoting rhizobacteria as bio-fertilizers. Plant Soil, 255: 571-586.

Zhang, X., Hause, R.J., Borevitz, J.O. 2012. Natural genetic variation for growth and development revealed by high-throughput phenotyping in Arabidopsis thaliana. G3 2: 29-34.

\section{How to cite this article:}

Areej Ali Baeshen. 2016. Use of Pseudomonas aeruginosa as Fertilizer in Eruca sativa. Int.J.Curr.Microbiol.App.Sci. 5(10): 301-312. doi: http://dx.doi.org/10.20546/ijcmas.2016.510.034 